\title{
Kidney function is associated with severity of white matter hyperintensity in patients with acute ischemic stroke/TIA
}

\author{
Lixia Zong ${ }^{1}$, Ming Yao ${ }^{1}$, Jun $\mathrm{Ni}^{1}$, Lixin Zhou', Jing Yuan ${ }^{1}$, Bin Peng ${ }^{1}$, Yi-Cheng Zhu ${ }^{1 *+}$ and Liying Cui ${ }^{1,2^{*+}}$
}

\begin{abstract}
Background: Previous studies suggested the potential interactions between cerebrovascular diseases and impaired renal function. However, the relationship between renal function and white matter hyperintensity (WMH), marker of cerebral small vessel disease, in patients with acute ischemic stroke (AIS) or transient ischemic attack (TIA) remains unknown.

Methods: We consecutively enrolled 1632 subjects with AIS or TIA who underwent brain MRI for this analysis. The severity of WMH in both of periventricular (PVH) and deep subcortical white matter (SDWMH) was evaluated using Fazekas scale. Estimated glomerular filtration rate (eGFR) was calculated by the equation of the Modification Diet for Renal Disease. Multinomial logistic regression was performed to evaluate the association between the severity of WMH and eGFR.
\end{abstract}

Results: Advanced age and hypertension were independently associated with the severity of both $\mathrm{PVH}$ and SDWMH (all $p<0.001$ ). There is a significantly inverse association between eGFR and PVH. Patients having each $30 \mathrm{ml} / \mathrm{min} / 1.73 \mathrm{~m}^{2}$ increase in eGFR was associated with $75 \%$ of risk of having degree 3 of WMH in periventricular areas compared with degree $0(p=0.04, \mathrm{OR}=0.75,95 \% \mathrm{Cl} 0.61-0.92)$. However this inverse association was not found between eGFR and SDWMH ( $P=0.50, \mathrm{OR}=0.93,95 \% \mathrm{Cl} 0.75-1.14)$.

Conclusion: Our study demonstrates that renal dysfunction (eGFR) is independently associated with the severity of PVH but not SDWMH in patients with acute ischemic stroke. This results highlighted different pathological mechanism and risk factors of $\mathrm{PVH}$ and SDWMH.

Keywords: Renal dysfunction, White matter hyperintensity, Chronic kidney disease, Stroke

\section{Background}

Chronic kidney disease has been recognized as a rapidly growing global health burden in the past decade. Previous studies showed that individuals with an eGFR below $60 \mathrm{~mL} / \mathrm{min}$ per $1.73 \mathrm{~m}^{2}$ had a higher risk of stroke and vice versa, suggesting the potential interactions between cerebrovascular diseases and impaired renal function $[1,2]$.

The regulation of the microvasculatures of brain and kidney is functionally similar. In addition, kidney impairment is characterized by glomerular endothelial dysfunction and

\footnotetext{
* Correspondence: zhuych910@163.com; pumchcuily@sina.com

${ }^{\dagger}$ Equal contributors

${ }^{1}$ Department of Neurology, Peking Union Medical College Hospital, Chinese

Academy of Medical Sciences, No.1 Shuaifuyuan, Beijing 100730, China

Full list of author information is available at the end of the article
}

lipohyalinosis, both of which are features of smallartery diseases [1, 3]. Thus people can readily presume that there might be association between kidney function and silent MRI changes which related to cerebral small vessel disease, like white matter hyperintensity (WMH), lacune and microbleeds. Previous studies showing independent associations between chronic kidney disease (CKD) and WMH in individuals without stroke supported the above mentioned hypothesis [4-6]. However, data on stroke patients have been subjected to debate $[7,8]$. WMH burden was related to increased risk of stroke and unfavorable post-stroke outcomes, so was impaired renal function [9-11]. Therefore the relationship between WMH and renal failure in ischemic stroke patients still needs more investigations. Moreover, although 
WMH in periventricular area and in deep white matter were regarded to have different pathological features [12], studies evaluated the lesions respectively in two locations were rare.

In the present study based on data collected in a large cohort of Chinese patients with acute ischemic stroke (AIS) or transient ischemic attack (TIA), we aimed to explore the potential risk factors of WMH, particularly the association between renal function and WMH.

\section{Methods \\ Patients}

Data were obtained from the SMART study, a multicenter trial designed to assess the effectiveness of a guideline-based structured care program for secondary stroke prevention in China. The complete study protocol, approved by the ethics committees at Peking Union Medical College Hospital, has been detailed elsewhere [13] and the reference number is S-151. Informed written consent was obtained from each patient.

Briefly, between April 2008 and December 2010, a total of 3821 patients, aged $>18$ years, with cerebral ischemic infarct or TIA within 30 days were enrolled. Patients who had severe comorbidities including heart failure, respiratory failure, renal failure, severe liver dysfunction, malignancy were excluded. For this study, only the 1975 patients who had brain MRI examination were included. 233 scans were excluded because of motion artifacts, leaving 1752 patients for WMH evaluation. Among patients who had WMH evaluation, those who had missing data on cardiovascular risk factors were further excluded so that the final sample was composed of 1632 subjects.

\section{Definition of risk factors}

A history of ischemic heart disease (IHD) was considered if a history of myocardial infarction, bypass cardiac surgery, or angioplasty was recorded. Atrial fibrillation (AF) was considered according to the electrocardiogram manifestation on admission or previously documented diagnosis. A history of ischemic stroke/TIA was considered according to previously documented diagnosis.

Diabetes mellitus (DM) was considered present when fasting blood glucose level $\geq 7.0 \mathrm{mmol} / \mathrm{L}$, or antidiabetic drugs were taken or a current history of DM was reported. Hypertension was defined by high blood pressure (systolic blood pressure $\geq 140 \mathrm{mmHg}$ or diastolic blood pressure $\geq 90 \mathrm{mmHg}$ ), or by use of antihypertensive drugs, or previous diagnosis. Hypercholesterolemia was defined as total cholesterol $\geq 5.2 \mathrm{mmol} / \mathrm{L}$ or lipid-lowing drugs were taken or a current history was documented. Smoking habits were categorized as nonsmokers and smokers (former or current). Admission serum creatinine was abstracted from the medical chart. Estimated glomerular filtration rate (eGFR) was calculated using the equation of the Modification Diet for Renal Disease modified by the Chinese coefficient [14]: eGFR $\left(\mathrm{ml} / \mathrm{min} / 1.73 \mathrm{~m}^{2}\right)=186 \times$ serum creatinine $(\exp [-1.154]) \times$ age $\quad(\exp [-0.203]) \times 1.233 \times 0.742 \quad$ if female). According to the eGFR, CKD stages were defined as follow: stage $1: e G F R \geq 90 \mathrm{ml} / \mathrm{min}$ per $1.73 \mathrm{~m}^{2}$, stage $2: 60 \leq \mathrm{eGFR}<90 \mathrm{ml} / \mathrm{min}$ per $1.73 \mathrm{~m}^{2}$, stage 3 : $30 \leq \mathrm{eGFR}<60 \mathrm{ml} / \mathrm{min}$ per $1.73 \mathrm{~m}^{2}$, stage $4: 15 \leq \mathrm{eGFR}$ $<30 \mathrm{ml} / \mathrm{min}$ per $1.73 \mathrm{~m}^{2}$, stage5: eGFR $<15 \mathrm{ml} / \mathrm{min}$ per $1.73 \mathrm{~m}^{2}[15]$.

\section{Rating of WMH}

The brain MRI scan had been performed on a 1.5-Tesla or 3.0-Tesla System due to different research centers, then MRI scans were converted from DICOM to analysis format by eZdicom software, and T2 fluid-attenuated inversion recovery (FLAIR) sequences were used to evaluate the degree of WMH. The Fazekas scale [16] was used to score both the severity of periventricular WMH (PVH) and deep subcortical WMH (SDWMH). All images were analyzed by the same experienced reader (X.-F.L.) blinded to all clinical data. The intra-rater agreement for the rating of WMH was assessed on a random sample of 54 subjects at 8-week intervals. The intrarater reliability analysis showed a good reliability with $\mathrm{k}$ values of 0.73 and 0.79 for PVH and SDWMH respectively.

\section{Statistical analysis}

The SPSS Version 19.0 was used for all analyses. The descriptive statistics on the baseline characteristics are presented as well as their crude distribution according to WMH degrees. The continuous variables were summarized as mean $\pm \mathrm{SD}$ or median with interquartiles, and all the categorical variables were presented as number (percent). Univariate analysis was used to evaluate the relationship between WMH and other variables (data not shown), and a nominal $P$ value less than 0.2 was used to select variables to build the multinomial regression model. For multinomial logistic regression, with both $\mathrm{PVH}$ and SDWMH rated with a 4-degree score as the dependent variable, each response category was contrasted against the reference category (degree 0). Each model adjusted on age, gender and hypertension at least. Statistical significance level for all analyses was set at $P$ value less than 0.05 .

\section{Results}

Baseline characteristics of the study sample are shown in Table 1 . The mean age was 62.3 years $(\mathrm{SD}=11.5)$ and 1118 (68.5\%) subjects were male. As the patients with severe comorbid illness had been excluded on admission, there are few people with CKD of stage 4 and 5 ( 5 for stage 4 and none for stage 5), and only 59 (3.6\%) 
Table 1 Baseline characteristics of the participants

\begin{tabular}{|c|c|}
\hline \multirow[t]{2}{*}{ Characteristic } & Total \\
\hline & N (\%) \\
\hline Age, years* & $62.3 \pm 11.5$ \\
\hline Male gender & 1118 (68.5 \%) \\
\hline Smoking & $776(47.6 \%)$ \\
\hline Diabetes mellitus & 508 (31.1\%) \\
\hline Hypertension & 1372 (84.1%) \\
\hline Hypercholesterolemia & $723(44.3 \%)$ \\
\hline Serum creatinine, umol/L & $77.4(65.9-90.0)$ \\
\hline $\mathrm{eGFR}, \mathrm{ml} / \mathrm{min} / 1.73 \mathrm{~m}^{2}$ & $106.9(89.3-127.9)$ \\
\hline Atrial fibrillation & $69(4.2 \%)$ \\
\hline Ischemic heart disease & $187(11.5 \%)$ \\
\hline History of ischemic stroke/TIA & $434(26.6 \%)$ \\
\hline \multicolumn{2}{|l|}{ CKD stages } \\
\hline Stage 1 & $1211(74.2 \%)$ \\
\hline Stage 2 & $362(22.2 \%)$ \\
\hline Stage 3 & $54(3.3 \%)$ \\
\hline Stage 4 & $5(0.3 \%)$ \\
\hline Stage 5 & $0(0 \%)$ \\
\hline \multicolumn{2}{|l|}{ PVH degrees } \\
\hline Degree 0 & $293(18.0 \%)$ \\
\hline Degree 1 & 749 (45.9 \%) \\
\hline Degree 2 & $416(25.5 \%)$ \\
\hline Degree 3 & $174(10.7 \%)$ \\
\hline \multicolumn{2}{|l|}{ SDWMH degrees } \\
\hline Degree 0 & $811(49.7 \%)$ \\
\hline Degree 1 & $575(35.2 \%)$ \\
\hline Degree 2 & $131(8.0 \%)$ \\
\hline Degree 3 & $115(7.0 \%)$ \\
\hline
\end{tabular}

Variables are presented as mean $\pm \mathrm{SD}(*)$, median (first-third quartile) or number (percentage)

Abbreviations: eGFR estimated glomerular filtration rate, CKD chronic kidney disease, $P V H$ periventricular white matter hyperintensity, SDWMH subcortical deep white matter hyperintensity

patients had an eGFR below $60 \mathrm{ml} / \mathrm{min}$ per $1.73 \mathrm{~m}^{2}$ (Total of CKD stage 3, 4 and 5).

Of 1632 participants, 811(49.7\%) had no WMH (degree 0 ) in the subcortical area, 575 (35.2\%) had SDWMH of degree 1, leaving only 246 (15\%) with SDWMH of degree 2 or 3 . By contrast, only 293 (18.0\%) participants had no visible WMH in the periventricular area, 749 (45.9\%) participants had PVH of degree 1, 590 (36.2 \%) had PVH of degree 2 or 3 (Table 1 ).

\section{Renal function and other factors associated with the severity of WMH}

The baseline distribution of potential risk factors in relation to WMH degrees and their associations with $\mathrm{PVH}$ as well as SDWMH are respectively shown in Tables 2 and 3. Mean age increased with WMH degree in both brain locations (Table 2); each SD increase in age was associated with a higher odds of having higher degrees of WMH. This effect of age is more obvious in $\mathrm{PVH}$ than in SDWMH, particularly in degree $3(\mathrm{OR}$ for $\mathrm{PVH}$ : 6.98, 95 \% CI 5.27-9.25; for SDWMH: 3.45, 95 \% CI 2.63-4.53).

The proportion of individuals with hypertension tended to increase with the degree of WMH; Similarly, subjects with hypertension tended to have higher odds of having higher degrees of PVH and SDWMH $(P<$ 0.0001 for both PVH and SDWMH) as compared with normotensive subjects (Tables 2 and 3 ).

We also observed that the median serum creatinine level increased with WMH degree and median GFR decreased while the degree of WMH increased (Table 2). After adjusted on age, gender and hypertension, patients having each $30 \mathrm{ml} / \mathrm{min} / 1.73 \mathrm{~m}^{2}$ increase in eGFR was associated with $75 \%$ of risk of having degree 3 of WMH in periventrical areas as compared with having degree 0 . However, this inverse association was not found when look at WMH in deep white matter (OR: 0.93, $95 \% \mathrm{CI}$ $0.75-1.14 ; P=0.50$, Table 3$)$. There was no obvious change after further adjustment on diabetes mellitus, hypercholesterolemia, history of ischemic heart disease and ischemic stroke/TIA. (Model 2, data not shown).

No significant associations were observed between WMH and gender, smoking status, diabetes, hypercholesterolemia, history of atrial fibrillation. Subjects with history of ischemic heart disease had higher risk of SDWMH $(P=0.05$, Table 3$)$, especially SDWMH of degree2 versus degree 0 (OR:1.68, 95 \% CI 1.01-2.79, Table 3), but after further adjustment for diabetes mellitus, hypercholesterolemia and eGFR, the value of 1.0 was included in the confidence interval (Model 2, data not shown). Subjects with history of ischemic stroke/ TIA had more WMH in both periventricular and subcortical area $(P<0.001$, Table 3$)$, especially the highest degree(For PVH, OR: 2.58, 95 \% CI 1.62-4.11; For SDWMH, OR:2.35, 95 \% CI 1.53-3.59, Table 3). Further adjustment for diabetes mellitus, hypercholesterolemia and eGFR did not change the ORs obviously.

\section{Discussion}

This study, performed in a large cohort of patients with AIS/TIA, found that renal function, as measured by eGFR, is inversely associated with WMH in periventrical areas. This association was independent of age, hypertension and other vascular risk factors. However, no association was found between eGFR and WMH in subcortical deep white matter.

The association between decreased GFR and increased $\mathrm{WMH}$ is in line with a study of 378 patients with 
Table 2 Baseline distribution of potential risk factors across the WMH degrees

\begin{tabular}{|c|c|c|c|c|c|c|c|c|}
\hline & \multicolumn{4}{|l|}{$\mathrm{PVH}$} & \multicolumn{4}{|l|}{ SDWMH } \\
\hline & Degree0 & Degree 1 & Degree 2 & Degree 3 & Degree0 & Degree 1 & Degree 2 & Degree 3 \\
\hline & $n=293$ & $n=749$ & $n=416$ & $n=174$ & $n=811$ & $n=575$ & $n=131$ & $n=115$ \\
\hline Age, mean years(SD) & $53.6(10.3)$ & $61.5(10.4)$ & $65.9(10.6)$ & $71.7(8.3)$ & $58.4(11.0)$ & $64.7(10.8)$ & $67.7(9.7)$ & $71.3(8.8)$ \\
\hline Male gender & 221 (75.4 \%) & $490(65.4 \%)$ & $283(68.0 \%)$ & $124(71.3 \%)$ & $584(72.0 \%)$ & 386 (67.1 \%) & $81(61.8 \%)$ & $67(58.3 \%)$ \\
\hline Smoking & $170(58.0 \%)$ & $343(45.8 \%)$ & $186(44.7 \%)$ & 77 (44.3\%) & $417(51.4 \%)$ & $268(46.6 \%)$ & $53(40.5 \%)$ & $38(33.0 \%)$ \\
\hline Diabetes mellitus & 77 (26.3 \%) & $260(34.7 \%)$ & $125(30.0 \%)$ & $46(26.4 \%)$ & $242(29.8 \%)$ & 201 (35.0 \%) & $33(25.2 \%)$ & $32(27.8 \%)$ \\
\hline Hypertention & $211(72.0 \%)$ & $635(84.8 \%)$ & $366(88.0 \%)$ & $160(92.0 \%)$ & $640(78.9 \%)$ & $508(88.3 \%)$ & $120(91.6 \%)$ & $104(90.4 \%)$ \\
\hline Hypercholesterolemia & $131(44.7 \%)$ & 354 (47.3 \%) & $175(42.1 \%)$ & $63(36.2 \%)$ & $360(44.4 \%)$ & $263(45.7 \%)$ & $62(47.3 \%)$ & $38(33.0 \%)$ \\
\hline Serum creatinine, umol/L & $\begin{array}{l}75.0 \\
(63.9-85.3)\end{array}$ & $\begin{array}{l}77.0 \\
(65.0-90.0)\end{array}$ & $\begin{array}{l}78.1 \\
(66.0-92.8)\end{array}$ & $\begin{array}{l}84.1 \\
(71.0-96.0)\end{array}$ & $\begin{array}{l}76.0 \\
(65.0-88.0)\end{array}$ & $\begin{array}{l}79.0 \\
(66.0-92.0)\end{array}$ & $\begin{array}{l}79.1 \\
(67.1-92.0)\end{array}$ & $\begin{array}{l}80.0 \\
(65.0-92.0)\end{array}$ \\
\hline eGFR, ml/min/1.73 m² & $\begin{array}{l}118.7 \\
(101.9-135.9)\end{array}$ & $\begin{array}{l}107.3 \\
(89.4-129.5)\end{array}$ & $\begin{array}{l}102.9 \\
(86.3-123.0)\end{array}$ & $\begin{array}{l}99.0 \\
(81.0-112.1)\end{array}$ & $\begin{array}{l}112.2 \\
(93.7-132.3)\end{array}$ & $\begin{array}{l}102.9 \\
(87.1-123.5)\end{array}$ & $\begin{array}{l}98.6 \\
(85.4-115.4)\end{array}$ & $\begin{array}{l}98.7 \\
(83.8-114.8)\end{array}$ \\
\hline Atrial fibrillation & $7(2.4 \%)$ & $30(4.0 \%)$ & $21(5.0 \%)$ & $11(6.3 \%)$ & $33(4.1 \%)$ & $19(3.3 \%)$ & $6(4.6 \%)$ & $11(9.6 \%)$ \\
\hline Ischemic heart disease & $22(7.5 \%)$ & $72(9.6 \%)$ & $67(16.1 \%)$ & 26 (14.9\%) & $72(8.9 \%)$ & 64 (11.1\%) & $27(20.6 \%)$ & $24(20.9 \%)$ \\
\hline Ischemic stroke/TIA & $53(18.1 \%)$ & $176(23.5 \%)$ & $126(30.3 \%)$ & 79 (45.4 \%) & $171(21.1 \%)$ & $173(30.1 \%)$ & $39(29.8 \%)$ & $51(44.3 \%)$ \\
\hline
\end{tabular}

ischemic stroke, which reported that eGFR $<60 \mathrm{~mL} /$ $\mathrm{min} / 1.73 \mathrm{~m}^{2}$ was associated with severe WMLs independent of age and sex [8]. Most studies in the general population also found this similar association. These results suggested that kidney impairment might serve as a predictive marker for the presence of white matter lesions. However, our finding is in contrast with another study of 523 subjects with acute ischemic stroke which reported no significant association between eGFR and WMH volume [7]. The discrepancy may be explained by the varied definitions of clinical characteristics and the different confounders corrected for from study to study. On the other hand, stroke patients may have more risk factors and more complicated pathogenesis underlying
WMH burden accumulation than general population, so there are more controversies about the correlation between GFR and WMH in patients with acute ischemic stroke.

We also found in our sample that the severity of both $\mathrm{PVH}$ and SDWMH were significantly associated with age, hypertension and a history of ischemic stroke/TIA. This result is consistent with previous studies showing that WMH burden is greater in individuals with common cerebrovascular risk factors [17]. Though these vascular risk factors are also predictors for GFR decline [18], we cannot determine whether the association between declined GFR and WMH observed in our study is common consequence from shared risk factors or

Table 3 Associations between risk factors and WMH degrees

\begin{tabular}{|c|c|c|c|c|c|c|c|c|}
\hline \multirow[t]{3}{*}{$N=1632$} & \multicolumn{4}{|l|}{$\mathrm{PVH}$} & \multicolumn{4}{|l|}{ SDWMH } \\
\hline & \multicolumn{4}{|l|}{ OR $(95 \% \mathrm{Cl})$} & \multicolumn{4}{|l|}{ OR $(95 \% \mathrm{Cl})$} \\
\hline & Degree 1 vs 0 & Degree 2 vs 0 & Degree 3 vs 0 & $P$ & Degree 1 vs 0 & Degree 2 vs 0 & Degree 3 vs 0 & $P$ \\
\hline$\overline{\mathrm{Age}^{a}}$ & $2.22(1.88-2.62)$ & $3.65(3.01-4.43)$ & $7.75(5.93-10.13)$ & $<0.001$ & $1.81(1.61-2.05)$ & $2.45(1.97-3.05)$ & $3.83(2.96-4.95)$ & $<0.001$ \\
\hline Male gender & $0.79(0.57-1.09)$ & $1.06(0.73-1.53)$ & $1.51(0.94-2.41)$ & 0.004 & $0.95(0.75-1.22)$ & $0.82(0.55-1.22)$ & $0.77(0.50-1.17)$ & 0.56 \\
\hline Hypertension & $1.96(1.39-2.76)$ & $2.56(1.68-3.92)$ & $4.06(2.12-7.80)$ & $<0.001$ & $1.88(1.37-2.58)$ & $2.65(1.38-5.08)$ & $2.27(1.16-4.43)$ & $<0.001$ \\
\hline eGFR ${ }^{b}$ & $0.89(0.78-1.01)$ & $0.92(0.80-1.06)$ & $0.75(0.61-0.92)$ & 0.04 & $0.96(0.87-1.07)$ & $0.87(0.72-1.06)$ & $0.93(0.75-1.14)$ & 0.50 \\
\hline Diabetes mellitus & $1.25(0.91-1.72)$ & $0.98(0.68-1.40)$ & $0.84(0.52-1.33)$ & 0.08 & $1.16(0.91-1.47)$ & $0.71(0.46-1.10)$ & $0.83(0.53-1.31)$ & 0.09 \\
\hline Hypercholesterolemia & $1.00(0.75-1.34)$ & $0.85(0.61-1.19)$ & $0.76(0.50-1.17)$ & 0.37 & $1.05(0.84-1.32)$ & $1.14(0.77-1.67)$ & $0.66(0.43-1.02)$ & 0.16 \\
\hline Smoking & $0.83(0.58-1.20)$ & $0.85(0.56-1.28)$ & $0.97(0.58-1.61)$ & 0.70 & $1.12(0.85-1.47)$ & $1.03(0.65-1.65)$ & $0.85(0.51-1.41)$ & 0.70 \\
\hline Atrial fibrillation & $1.00(0.42-2.39)$ & $1.02(0.40-2.60)$ & $1.05(0.36-3.01)$ & 1.00 & $0.56(0.31-1.01)$ & $0.67(0.27-1.69)$ & $1.23(0.57-2.65)$ & 0.12 \\
\hline Ischemic heart disease & $0.69(0.41-1.17)$ & $1.04(0.60-1.80)$ & $0.80(0.41-1.54)$ & 0.15 & $0.91(0.63-1.31)$ & $1.68(1.01-2.79)$ & $1.52(0.89-2.61)$ & 0.05 \\
\hline Ischemic stroke/TIA & $1.09(0.76-1.56)$ & $1.42(0.96-2.10)$ & $2.58(1.62-4.11)$ & $<0.001$ & $1.36(1.06-1.76)$ & $1.27(0.83-1.94)$ & $2.35(1.53-2.59)$ & 0.001 \\
\hline
\end{tabular}

Models of multinomial logistic regression adjusted on age, gender and hypertension. In each model, PVH or SDWMH was considered as the dependent variable categorized in 4 degrees (0-3), and degree 0 was the reference category

${ }^{\mathrm{a}}$ For age, the OR estimates the association related to an increase of $1 \mathrm{SD}$

${ }^{b}$ For eGFR, the OR estimates the association related to increase of $30 \mathrm{ml} / \mathrm{min} / 1.73 \mathrm{~m}^{2}$ 
represents a causative relationship, considering our research design.

In our study, the severity of WMH was evaluated separately in two areas and those which located in deep white matter was found not relate to renal function. Our findings have suggested distinct involvement of renal function in the process of WMH accumulation in two locations. Previous studies have also reported that different mechanism involved in the development of $\mathrm{PVH}$ and SDWMH, and PVHs are more likely due to diminished cerebral vasomotor reactivity and subsequent hypoperfusion, while SDWMHs are related to microangiopathy $[12,19,20]$. However, only a few studies detected the correlation of GFR with PVH and SDWMH separately. A recent study which distinguished the relationship of GFR with $\mathrm{PVH}$ and SDWMH in a large cohort of patients without stroke history showed that eGFR $<60 \mathrm{ml} / \mathrm{min} / 1.73 \mathrm{~m}^{2}$ is significantly associated with both PVH and SDWMH [21]. In contrast with our study, they targeted population without history of stroke or other cardiovascular events, and the proportion of subjects with potential risk factors in their study is low, thus the contribution of renal dysfunction to WMH may be augmented.

There are several limitations should be noted in our study. First, considered that it was a multicenter designed study and the serum creatinine concentration had been analyzed in different laboratories, there may be bias of measurement. However, the comparability of serum creatinine concentration detected in different lab was widely accepted in previous studies, and all of the involved centers have rigid quality control process of their clinical and biochemical tests, we think this may not change our results. Second, the WMH assessment had been performed using visual rating scale. Although also widely used, visual rating may be less precise or consistent than quantitative evaluation. Last, as we have excluded subjects with severe renal dysfunction, the proportion of patients with eGFR $<60 \mathrm{ml} / \mathrm{min} / 1.73 \mathrm{~m}^{2}$ is lower (only $3.6 \%$ ) in our cohort than previous reported $(28.1 \%$ in one study and $40.2 \%$ in another) $[8,22]$, which may underestimate the association of eGFR over WMH.

\section{Conclusion}

In conclusion, we demonstrated that kidney function is associated with WMH in patients having acute ischemic stroke or TIA. Furthermore, this association was found only in WMH located around ventricles. Further studies are needed to improve our understanding of this cerebro-renal coexisting impairment.

\section{Abbreviations}

AF: Atrial fibrillation; AIS: Acute ischemic stroke; CKD: Chronic kidney disease; DM: Diabetes mellitus; eGFR: Estimated glomerular filtration rate; IHD: Ischemic heart disease; PVH: Periventricular white matter hyperintensity; SDWMH: Deep subcortical white matter hyperintensity; TIA: Transient ischemic attack; WMH: White matter hyperintensity; WMH: White matter hyperintensity

\section{Acknowledgements}

We are grateful to SMART participants and our colleagues in the SMART study for data collection and management.

\section{Funding}

This study was supported by the National Natural Science Foundation of China (grant number: 81173663), and National Key Technology R\&D Program in the 11th and 12th five-year Plan of China (grant number: 2006BA101A10, 2011BAI08B03).

\section{Availability of data and materials}

The dataset supporting the conclusions of this article is not available in the public repository. We are willing to share the dataset if any researcher asks.

\section{Authors' contributions}

LXZ participated in the experiments, analyzed data, and drafted the manuscript. MY helped to analyze data and draft the manuscript. JN participated in the acquisition of data. LXZ participated in the acquisition of data. JY participated in the data analysis. BP participated in interpretation of data. YCZ conceived the study, participated in its design, interpretation of data and helped to draft the manuscript. LYC conceived the study, participated in its design. All authors read and approved the final manuscript.

\section{Competing interests}

The authors declare that they have no competing interests.

\section{Consent for publication}

Not applicable.

\section{Ethics approval and consent to participate}

The complete study protocol was approved by the ethics committees at Peking Union Medical College Hospital with the reference number S-151. Informed written consent was obtained from each patient.

\section{Author details}

1Department of Neurology, Peking Union Medical College Hospital, Chinese Academy of Medical Sciences, No.1 Shuaifuyuan, Beijing 100730, China. ${ }^{2}$ Department of Neurology, Peking Union Medical College Hospital, Neuroscience Center, Chinese Academy of Medical Sciences and Peking Union Medical College, No.1 Shuaifuyuan, Beijing 100730, China.

Received: 17 April 2016 Accepted: 29 September 2016

Published online: 06 October 2016

\section{References}

1. Toyoda K, Ninomiya T. Stroke and cerebrovascular diseases in patients with chronic kidney disease. Lancet Neurol. 2014;13:823-33.

2. Toyoda K. Cerebrorenal interaction and stroke. Contrib Nephrol. 2013;179:1-6.

3. Ito S, Nagasawa T, Abe M, Mori T. Strain vessel hypothesis: a viewpoint for linkage of albuminuria andcerebro-cardiovascular risk. Hypertens Res. 2009; 32:115-21.

4. Khatri M, Wright CB, Nickolas TL, Yoshita M, Paik MC, Kranwinkel G, et al. Chronic kidney disease is associated with white matter hyperintensity volume: the Northern Manhattan study (nomas). Stroke. 2007;38:3121-6.

5. Ikram MA, Vernooij MW, Hofman A, Niessen WJ, van der Lugt A, Breteler MM. Kidney function is related to cerebral small vessel disease. Stroke. 2008; 39:55-61.

6. Wada M, Nagasawa H, Iseki C, Takahashi Y, Sato H, Arawaka S, et al. Cerebral small vessel disease and chronic kidney disease $(\mathrm{ckd})$ : results of a crosssectional study in community-based Japanese elderly. J Neurol Sci. 2008; 272:36-42.

7. Rost NS, Rahman R, Sonni S, Kanakis A, Butler C, Massasa E, et al. Determinants of white matter hyperintensity volume in patients with acute ischemic stroke. J Stroke Cerebrovasc Dis. 2010;19:230-5.

8. Oksala NK, Salonen T, Strandberg T, Oksala A, Pohjasvaara T, Kaste M, et al. Cerebral small vessel disease and kidney function predict long-term survival in patients with acute stroke. Stroke. 2010;41:1914-20. 
9. Kim BJ, Lee SH. Prognostic impact of cerebral small vessel disease on stroke outcome. J Stroke. 2015;17:101-10.

10. Rowat A, Graham C, Dennis M. Renal dysfunction in stroke patients: a hospitalbased cohort study and systematic review. Int J Stroke. 2014;9:633-9.

11. Yeh SJ, Jeng JS, Tang SC, Liu CH, Hsu SP, Chen CH, et al. Low estimated glomerular filtration rate is associated with poor outcomes in patients who suffered a large artery atherosclerosis stroke. Atherosclerosis. 2015;239:328-34.

12. Fazekas F, Kleinert R, Offenbacher H, Schmidt R, Kleinert G, Payer F, et al. Pathologic correlates of incidental mri white matter signal hyperintensities. Neurology. 1993;43:1683-9.

13. Peng B, Zhu Y, Cui L, Ni J, Xu W, Zhou L, et al. Standard medical management in secondary prevention of ischemic stroke in china (smart). Int J Stroke. 2011;6:461-5.

14. Ma YC, Zuo L, Chen JH, Luo Q, Yu XQ, Li Y, et al. Modified glomerular filtration rate estimating equation for chinese patients with chronic kidney disease. J Am Soc Nephrol. 2006;17:2937-44.

15. National Kidney F. K/dogi clinical practice guidelines for chronic kidney disease: evaluation, classification, and stratification. Am J Kidney Dis. 2002;39:S1-266.

16. Fazekas F, Chawluk JB, Alavi A, Hurtig HI, Zimmerman RA. Mr signal abnormalities at $1.5 \mathrm{t}$ in alzheimer's dementia and normal aging. AJR. Am J Roentgenol. 1987;149:351-6.

17. Jeerakathil T, Wolf PA, Beiser A, Massaro J, Seshadri S, D'Agostino RB, et al. Stroke risk profile predicts white matter hyperintensity volume: the Framingham study. Stroke. 2004;35:1857-61.

18. Ghaffar U, Maharjan N, Moore PC. Predictors of ckd and rate of decline in egfr in the elderly: a case-cohort study. Nephrol News Issues. 2016;30:38-45.

19. Schmidt R, Schmidt H, Haybaeck J, Loitfelder M, Weis S, Cavalieri M, et al. Heterogeneity in age-related white matter changes. Acta Neuropathol. 2011;122:171-85.

20. Abe A, Nishiyama Y, Harada-Abe M, Okubo S, Ueda M, Mishina M, et al. Relative risk values of age, acrolein, il-6 and crp as markers of periventricular hyperintensities: a cross-sectional study. BMJ Open. 2014;4:e005598.

21. Takahashi W, Tsukamoto Y, Takizawa S, Kawada S, Takagi S. Relationship between chronic kidney disease and white matter hyperintensities on magnetic resonance imaging. J Stroke Cerebrovasc Dis. 2012;21:18-23.

22. Yahalom G, Schwartz R, Schwammenthal Y, Merzeliak O, Toashi M, Orion D, et al. Chronic kidney disease and clinical outcome in patients with acute stroke. Stroke. 2009;40:1296-303.

\section{Submit your next manuscript to BioMed Central and we will help you at every step:}

- We accept pre-submission inquiries

- Our selector tool helps you to find the most relevant journal

- We provide round the clock customer support

- Convenient online submission

- Thorough peer review

- Inclusion in PubMed and all major indexing services

- Maximum visibility for your research

Submit your manuscript at www.biomedcentral.com/submit 